\title{
Evaluation of medication package inserts in Saudi Arabia
}

\author{
This article was published in the following Dove Press journal: \\ Drug, Healthcare and Patient Safety \\ 24 March 2012 \\ Number of times this article has been viewed
}

\author{
Sinaa A Al-aqeel \\ Clinical Pharmacy Department, \\ King Saud University, Riyadh, \\ Kingdom of Saudi Arabia
}

Correspondence: Sinaa Al-aqeel PO Box 3763 I6, Riyadh II335, Kingdom of Saudi Arabia Tel +966506 108592 Email salageel@ksu.edu.sa
Purpose: The aim of this study was to examine if package inserts (PIs) supplied with prescribed and over-the-counter medications in Saudi Arabia contain information relevant for the safe and appropriate use of these medications.

Methods: Sixty PIs for prescription-only medications $(\mathrm{n}=37)$ and over-the-counter medications $(n=23)$ were evaluated against a set of safety criteria compiled from the literature.

Results: Analyzed PIs were defective in many aspects. Particularly of concern were unclear dosage instructions, lack of measures to be taken when an administrative error was made, inappropriate presentation of side effects, and lack of measures to be taken if serious side effects occurred.

Conclusion: This study indicated that information relevant to the safe and appropriate use of medications was not uniformly mentioned in the PIs analyzed. To avoid medication errors due to deficits in the current PIs, we recommend improvement in the existing PIs based on best practice for information content and design.

Keywords: drug information, patient education, patient information leaflets, package inserts

\section{Introduction}

Written patient drug information, such as drug labels and package inserts (PIs), is intended to instruct patients on how and when to use a medicine and to promote an understanding of the purpose, benefits and risks of the medication prescribed. ${ }^{1}$ This understanding is supposed to lead to successful therapy and enable safe medication use. Substantial regulatory efforts have been made in Europe, the USA, and Australia to improve written drug information. ${ }^{1,2}$

In Saudi Arabia, during the pharmaceutical registration stage, manufacturers must submit a summary of product characteristics (SPC) and labeling information to the regulatory authority. The laws mandate that medications purchased from a community pharmacy are dispensed in their original packages with an insert very similar to the SPC. The intended users of PIs are health care professionals; however, because of their visibility one could expect that many patients will turn to PIs to answer their medication-related queries. Indeed, a Saudi-based survey of over 2000 community pharmacy customers found that $88 \%$ of respondents claimed that they read the PIs or ask somebody to read it for them. ${ }^{3}$ However, of concern is that the PIs could be the main source of information for some patients for many reasons. First, research suggests self-medication is common in Saudi Arabia. ${ }^{4}$ Second, purchasing legally prescription-only medications without a prescription from community pharmacies is common. ${ }^{5}$ This means that the patient assumes the bulk of responsibility for medication safety not only for over-the-counter 
(OTC) medications but also for medications such as antibiotics, nonsteroidal anti-inflammatories, and oral contraceptives. Third, even if patients visit physicians, information provided regarding dose and frequency, precautions, and adverse effects of their prescribed medications has been shown to be suboptimal, ${ }^{6}$ as has community pharmacists' counselling. ${ }^{7}$ Despite that, research on PIs in Saudi Arabia is rare. Available evidence indicates that inserts of Saudi-marketed drugs conveyed limited and incomplete information compared with their counterparts marketed in the $\mathrm{USA}^{8}$ and there is a substantial disagreement in information between the PIs of generic and brand products marketed in Saudi Arabia. ${ }^{9}$ However, no study has evaluated whether the available PIs are comprehensible and understandable by the patient, which is a very important aspect of evidence-based PIs. ${ }^{10} \mathrm{~A}$ growing body of research indicates that patients frequently misinterpret prescription drug labels and PIs. ${ }^{11-17}$ Challenges in reading and understanding labels and PIs may represent one cause of the high rates of medication errors and poor adherence. ${ }^{11,12}$ Therefore, this study aimed to assess the availability of key information on PIs supplied with prescribed and OTC medications in Saudi Arabia. It also assessed whether information on PIs is presented in a way that aids patient comprehension. In addition, characteristics of relevance to readability were examined.

\section{Methods PI selection}

A commercially available list of top-selling prescription-only and OTC medications in Saudi Arabia was obtained. ${ }^{18}$ From the list of 253 medicines, each of which had sold 100,000 units or more in Saudi during the year 2011, 60 medicines were selected. The 60 medications were chosen from the top 150 sold medications (prescription $=37$, OTC $=23$ ). Selection was done to ensure the drugs covered different therapeutic indications $(n=15)$, pharmaceutical forms (tablets, capsules, syrups, suspensions, drops, ointments, and inhalers), from different manufacturers ( $n=19$; four Saudi, two Arabic non-Saudi, 13 international). The legal status of each medication was verified by status information available on the Saudi Food and Drug Authority (SFDA) website. ${ }^{19}$ If a PI was for different strengths and one of these strengths was an OTC, the PI was counted with the OTC. The PIs were collected and analyzed by the author from community pharmacies in May 2011.

\section{Leaflet characteristics}

Six characteristics were examined: dimensions, layout, type of paper, color of paper and text, transparency, use of headings, and use of pictograms or graphics. Dimensions (length and width) of each PI were measured to the nearest centimeter with a standard ruler. Transparency was registered when print from the reverse side showed through the PI.

\section{Evaluation criteria}

PI content was evaluated using criteria derived from the literature - specifically, the criteria used by Fuchs et $\mathrm{al}^{20}$ and Raynor et al. ${ }^{2}$ Fuchs et al's criteria were built on European and German regulations. Many of these quality criteria referred to the content of the PI, such as contraindications, and the dosage or possible side effects. There were also some criteria that considered additional aspects such as general comprehensibility and readability as well as layout. These criteria had already been used to analyze German PIs ${ }^{11}$ and were tested in the PI test study (PAINT) study, ${ }^{13}$ which showed that they significantly improved comprehensibility and usability of PIs. ${ }^{12}$ The criteria used by Raynor and colleagues was based on the US Keystone Criteria, ${ }^{2}$ which were developed through a structured process of consensus by a large number of stakeholders. Criteria related to readability, such as word count and font size, were excluded as they were outside the objective of this study.

Evaluations were made on a "yes" or "no" basis with "yes" meaning the information was provided and "no" meaning no such information was provided.

\section{Data analysis and presentation}

The data were extracted by the author twice at different times to minimize the chances of missing any information. The number of PIs that met the quality criteria was calculated. The results were expressed as absolute numbers and percentages. The Chi-square test was conducted to determine any significant differences among groups. Statistical significance was determined at a $P$ value lower than 0.05 . All statistical analysis was performed using SPSS (v 15.00; IBM Corporation, Armonk, NY).

\section{Results Leaflet characteristics}

All PIs use uncoated paper, but in all PIs the paper used was not sufficiently thick to reduce transparency. All analyzed PIs used a white background with text in black $(n=51,[85 \%])$, blue $(n=7,[12 \%])$, or red $(n=2,[3 \%])$. Arabic translation was available in 58 (97\%). PI headings were used in all PIs. Twenty (33\%) analyzed PIs were in landscape format. The mean length of the pages on which the PIs was printed was $26.6 \mathrm{~cm}$ (standard deviation [SD] 14.7), while the average 
width of pages was $20 \mathrm{~cm}$ (SD 10.9). Five (8\%) PIs used images, pictograms, or other graphics. Abbreviations and acronyms other than "mg," "mL," or "kg" were used in 21 (35\%) PIs. Scientific symbols (such as " $>$ " or " $<$ ") were used in 19 (32\%) PIs. The explanation for medical terminology was available in eight (13\%) PIs. Forty-four PIs (73\%) included information on the last version update. The date of last update in 26 (65\%) PIs was between 2005 and 2009. The intended users of the leaflet were specified in $10 \%$ of PIs ( $n=6$; two for health care professionals, four for patients). However, the text targeted particular patients in another seven (11\%) PIs, evident from the use words such as "your medication," "inform your doctor," or "if you notice any side effects."

All the PIs included the manufacturer's name; however, four PIs (7\%) did not include the manufacturer's address.
Approximately $82 \%(\mathrm{n}=49)$ provided information on correct storage temperature.

\section{Information provided}

All PIs contained information on name, active ingredients, therapeutic indications, and pharmaceutical forms. Thirty-two (53\%) PIs were for more than one strength and/ or different pharmaceutical forms. Although all PIs provided dosage instructions, precautions and adverse effects information, many lacked important information (Table 1). The route of administration was not explicitly indicated, such as "oral route" or "swallow the tablet," in 24 (40\%) analyzed PIs (15 OTC, nine prescription). Dosage instructions were given as number of tablets or capsules, as volume, drops, or amount of the drug instead of in milligrams of active

Table I Package inserts $(n=60)$ content evaluation criteria

\begin{tabular}{|c|c|c|c|c|c|c|}
\hline \multirow[t]{2}{*}{ Criteria } & \multicolumn{2}{|c|}{ OTC $^{a}$} & \multicolumn{2}{|c|}{ Prescription $^{\mathrm{b}}$} & \multicolumn{2}{|c|}{ Total } \\
\hline & $\mathbf{n}$ & $\%$ & $\mathbf{n}$ & $\%$ & $\mathbf{n}$ & $\%$ \\
\hline \multicolumn{7}{|l|}{ Dosage instructions } \\
\hline $\begin{array}{l}\text { All dosage instructions are given as number of tablets or capsules, as volume, } \\
\text { drops or amount of the drug instead of in milligrams of active substance }\end{array}$ & 13 & 57 & 19 & 51 & 32 & 53 \\
\hline \multicolumn{7}{|l|}{ an explanation are missing* } \\
\hline The maximum dose is included & 9 & 39 & 15 & 41 & 24 & 40 \\
\hline The maximum dose is given as number of tablets or capsules or as volume* & 6 & 26 & 2 & 5 & 8 & 13 \\
\hline Hints on period of use are available (duration of use) & 10 & 43 & 20 & 54 & 30 & 50 \\
\hline Hints on time of the day when the medicine should be used & 4 & 17 & 12 & 32 & 16 & 27 \\
\hline $\begin{array}{l}\text { Information such as take the medicine before, after, or independent of a meal } \\
\text { are available in case of orally taken drugss,* }\end{array}$ & 4 & 24 & 17 & 59 & 21 & 46 \\
\hline The type of solution to use is given for orally taken drugs with a solid application form ${ }^{c}$ & 5 & 29 & 6 & 21 & II & 18 \\
\hline The amount of solution to use is given by orally taken drugs with a solid application form c,* & 5 & 29 & 2 & 7 & 7 & 15 \\
\hline Hints that tablets and capsules should be taken in upright position & 0 & 0 & 0 & 0 & 0 & 0 \\
\hline Provides information on what to do in case of overdose of the medicine & 18 & 78 & 27 & 73 & 45 & 75 \\
\hline Provides information on what the patient should do if doses are missed* & 1 & 4 & 11 & 30 & 12 & 20 \\
\hline \multicolumn{7}{|l|}{ Precautions } \\
\hline Statement of contraindications & 22 & 96 & 36 & 97 & 58 & 97 \\
\hline Statement of drug interactions & 19 & 83 & 28 & 76 & 47 & 78 \\
\hline Information on laboratory, food, or herbal interactions & 3 & 13 & 9 & 25 & 12 & 20 \\
\hline Information on capability to drive a car or operate a machine & 8 & 35 & 18 & 49 & 26 & 43 \\
\hline Provides advice on when to consult a physician/pharmacist & 7 & 30 & 14 & 38 & 21 & 35 \\
\hline \multicolumn{7}{|l|}{ Adverse effects } \\
\hline Provides qualitative statements on the frequency of side effects (rare or common) & 14 & 61 & 24 & 65 & 38 & 63 \\
\hline $\begin{array}{l}\text { Verbal frequency terms are explained in the form of natural frequencies } \\
\text { (eg, very common "more than I in } 10 \text { patients") }\end{array}$ & 2 & 9 & 8 & 22 & 10 & 17 \\
\hline Describes severity of every possible adverse reaction & 3 & 13 & 5 & 14 & 8 & 13 \\
\hline Setting out the side effects by frequency of occurrence, starting with the highest & 2 & 9 & 15 & 41 & 17 & 28 \\
\hline Setting side effects by organ/system/class & 3 & 13 & 12 & 32 & 15 & 25 \\
\hline Statement on possible influence of the medication on reaction time* & 0 & 0 & 6 & 16 & 6 & 10 \\
\hline Describes suitable measures in case of adverse reactions* & 2 & 9 & 14 & 38 & 16 & 27 \\
\hline Possible side effects if medication is stopped or the dose is changed without doctor's advice & 0 & 0 & 5 & 14 & 5 & 8 \\
\hline
\end{tabular}

Notes: aOTC $n=23$ : Nonsteroidal anti-inflammatory $(n=7)$, anti-pyretic analgesic $(n=5)$, anti-histamine $(n=5)$, cough preparations $(n=2)$, anti-ulcer $(n=3)$, and vitamin

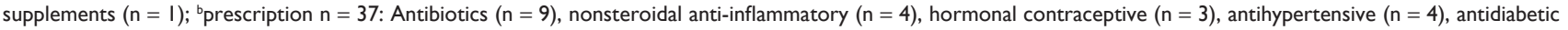
$(n=3)$, corticosteroids $(n=3)$, neurotropic/antiepileptic $(n=2)$, cough preparations $(n=2)$, antihypercholesterolemia $(n=2)$, anti-emetic $(n=2)$, anti-ulcer $(n=1)$, and haemorrhoids $(n=1)$; ${ }^{c}$ percentage calculated for oral preparation only $($ OTC $=17$, Prescription $=29)$. $* P<0.05$.

Abbreviation: OTC, over the counter. 
substance in $32(52 \%)$ PIs. Thirty PIs were for tablets or capsules and 16 PIs were for more than one pharmaceutical form, including tablets or capsules. The amount of solution to use with tablets or capsules was given in seven of the analyzed tablet or capsule PIs. Recommendations that tablets and capsules should be taken in an upright position were not provided in any of analyzed PIs.

Guidance on duration of use, such as "treatment should not be continued for more than four weeks without medical advice" was available in $30(50 \%)$ PIs. Statements on possible influence of the medication on reaction time, such as "this effect may appear after 5-7 days," were missing in 54 (90\%) PIs. Forty-four PIs (73\%) did not provide information on suitable measures in case of adverse reactions even general measures such as "contact your doctor or pharmacist."

There was no statistically significant difference between OTC and prescription-only drug PIs for most of the criteria (Table 1).

Information on use during pregnancy and nursing was available in $58(97 \%)$ and $52(87 \%)$ analyzed PIs, respectively (Figure 1). Twenty-two (38\%) PIs included information from animal studies on the safety or potential teratogencity. Instructions on possible use in children and the elderly could only be found in $44(73 \%)$ and 18 (30\%) PIs, respectively (Figure 1). Of these PIs, only $22(50 \%)$ provided information on children by age and five (28\%) PIs defined the age of the elderly.

\section{Discussion}

In this study, Saudi PIs were analyzed to assess if they contained information the patient could understand and follow to their best possible benefit. As Table 1 makes clear, PIs had many deficiencies. Particularly of concern was the lack of clarity in dosing instructions. Medication errors and adverse events in the outpatient setting have been linked to patients' unintentional misuse of prescribed or OTC medicines due to improper understanding of PI instructions, particularly in patients with limited literacy skills and those managing multiple medication regimens. ${ }^{11,12}$ Evidence suggests that patients are more likely to make an error if dosage instructions were given in milligrams of active substance instead of number of tablets. ${ }^{1-13}$ Patients are better able to interpret more explicit instructions, for example, "every 12 hours," compared with the more implicit "twice daily." 11,12 Also, their ability to determine the correct dose is reduced if presented with instructions such as "take 2-4 tablets." 13 Yet, approximately half of analyzed PIs presented dosage instructions this way. Evidence also suggests that when taking orally administered tablets, people should drink a minimum of $60 \mathrm{~mL}$ of liquid and position the upper body at a minimum angle of $45^{\circ}$ to achieve a high passage rate of tablets or capsules. ${ }^{21}$ Such instructions were missing in many of the analyzed PIs.

Of concern also is reporting of side effects. Very few PIs presented the likelihood of adverse effects using the recommended approach of verbal descriptions alongside natural frequencies, for example, "very common (more than 1 in 10 patients)." The use of verbal expressions alone or percentages are misunderstood and can lead to overestimation of the risk by the user, ${ }^{14,15}$ which may deter patients from taking their medication. ${ }^{22}$ Only $28 \%$ of analyzed PIs presented side effects grouped in terms of frequency and severity, which is more comprehensible for patients than organ systems. ${ }^{16}$

Patients are entitled to better information with their medication to enable them to make informed decisions as to whether to take the drug and to prepare them for what they

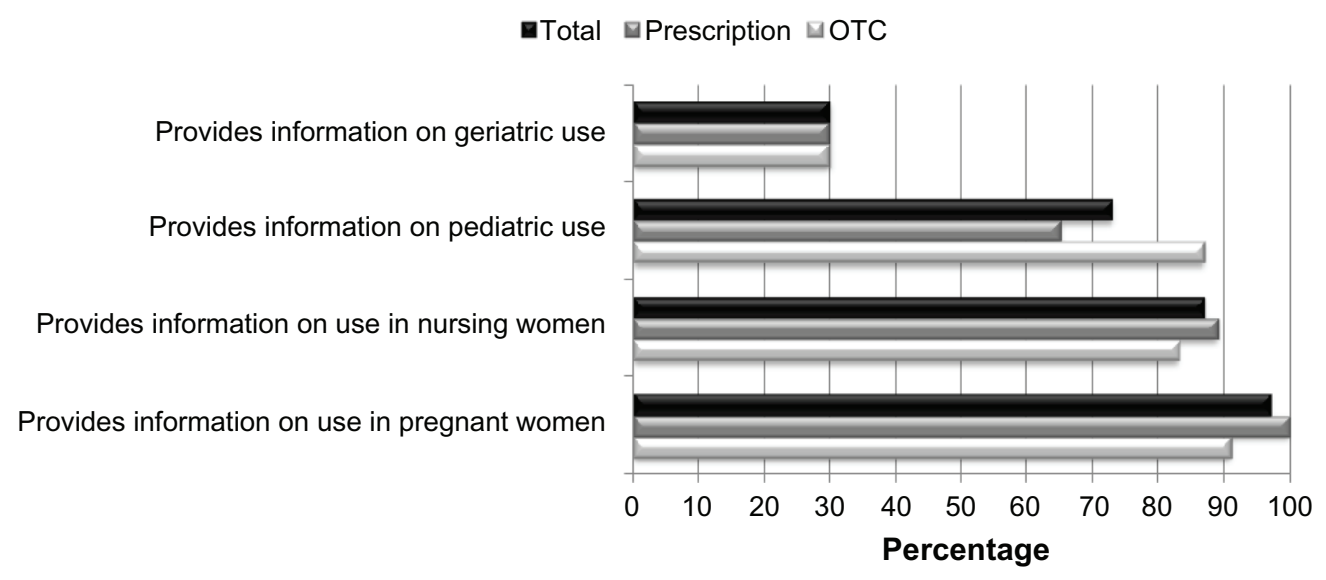

Figure I Package inserts $(n=60)$ content evaluation criteria with regard to use in pregnant, nursing, pediatric, and geriatric populations. Abbreviation: OTC, over the counter. 
might experience in response to a drug. ${ }^{1}$ However, analyzed PIs were often unclear about patient actions, such as what they should do if they miss a dose, what measures should be taken if serious side effects occur, and when to consult a doctor.

The problems identified in this study largely conform to those noted by others who examined patient information leaflets. ${ }^{2,15,20,23-27}$ An international research group compared five patient information leaflets from the UK with five from Australia and four from the USA. ${ }^{2}$ Leaflets from Australia showed the best quality, closely followed by those of the UK. The US leaflets were not only found lacking in provision of information about contraindications, precautions, and drug interactions, but also in readability and comprehensibility. ${ }^{2}$ An investigation of 68 German PIs from frequently used medicines ${ }^{20}$ found that many inserts lacked key safety information, including information on daily maximum dose and adverse effects, and were difficult to comprehend. Carrigan et $\mathrm{al}^{15}$ assessed the leaflets supplied with the 50 most frequently prescribed drugs in England and found that $20(40 \%)$ of the 50 leaflets gave no indication of the likelihood of adverse effects occurring and only four leaflets $(8 \%)$ provided any form of numerical indication of risk. Analysis of PIs accompanying marketed drug products in India $(n=80)$ found only five of the inserts had information on the most frequent adverse drug reactions associated with the drug. ${ }^{24}$ Also, information with regard to pediatric and geriatric use was present in only $44 \%$ and $13 \%$ of the PIs, respectively. Other studies on PIs for topical medications $(\mathrm{n}=15),{ }^{25}$ geriatric medications $(\mathrm{n}=50),{ }^{26}$ and antidepressants $(\mathrm{n}=42),{ }^{27}$ similarly found PIs lacking in adequate information about contraindications, precautions and drug interactions.

This study has three main limitations that should be considered when interpreting the results. First, a small number of PIs were analyzed. Second, PI samples were not collected randomly, which raises the possibility of selection bias. As a result, it may be possible that randomly choosing the PIs and increasing the sample size could lead to differences in the results found. However, considering the diversity of PIs examined with regards to indications, legal status, and manufacturers, other important findings are quite unlikely. Moreover, the number of PIs examined is in the range of sample size of previous similar research. Third, there are no gold standard criteria for evaluating written patient drug information ${ }^{28}$ and criteria used in this study, although used in previous research, have advantages and disadvantages.

Despite these limitations, this study represents the first attempt to evaluate PI adequacy as sources of information for patients in Saudi Arabia. The results presented here demonstrate the necessity and possibilities to improve PIs. Fortunately, from August 2011, a patient information leaflet (PIL) is now required to be submitted during the drug registration process. ${ }^{29}$ This does not mean, however, that the issues highlighted by this research will be fully resolved. It is not clear if or when PILs will replace the current PIs. Until the newly implemented PILs are widely available, most Saudi patients will receive no written information other than the PIs analyzed in this study. Furthermore, legislation and guidelines in other countries have not guaranteed that PIs are clear and easy to use. The SFDA guidelines state that PILs should be written "using patient understandable language," but provide no directions on what constitutes patient understandable language. Evidence suggests that specific content and format of prescription drug labels facilitate communication with and comprehension by patients. ${ }^{17,30} \mathrm{We}$ recommend, therefore, that the SFDA guideline demands the use of evidence-based best practices regarding how medication information should be written, designed, and delivered. Patient characteristics such as education and age have an effect on their understanding and usability of PIs. ${ }^{11,12,16,31}$ Thus, the SFDA should also require that PILs reflect the results of consultations with target patient groups, to understand fully the diverse needs of our patients. The mode of PI delivery, whether it is computer generated as in the USA, a PI as in Europe, or online via the SFDA website, also requires careful consideration.

The findings of this study should stimulate further research into the effects of content and format of PIs on readability, understanding, and medication use. The current study did not assess actual patient understanding of medication instructions; rather, it used criteria to measure comprehensibility and understandability. Research measuring actual patients' abilities to understand and act upon instructions provided in PIs is required. This paper has focused on the availability of key information and its comprehensibility, with less emphasis on the readability of PIs. Further research in this area is needed. The effect of alternative PI design and layout on comprehension among patients across all age groups and literacy levels also needs to be investigated. As the nature of the relationship between deficits in PIs and poor medication-taking behavior (ie, adherence) or unsafe use of medications has not been well tested, ${ }^{17}$ more studies evaluating the effects of PI content and format on adherence, medication error rates and health outcomes are needed. The design of these studies needs to capture the true complexity of medication-taking behavior, 
where patients may be taking multiple medications and have numerous competing demands.

\section{Conclusion}

This study indicates that information relevant to the safe and appropriate use of medications was not uniformly provided in the PIs analyzed. To avoid medication errors due to deficits in the current PIs, we recommend improvement in the existing PIs based on best practice for information content and design.

\section{Acknowledgments}

The help of Ahmed Bahatheq in providing the top selling medications list is very much appreciated. The author also thanks the research center at the Female Centre for Scientific and Medical Colleges, King Saud University, for their financial support towards the publication of this paper.

\section{Disclosure}

The author reports no conflicts of interest in this work.

\section{References}

1. Raynor DK, Blenkinsopp A, Knapp P, et al. A systematic review of quantitative and qualitative research on the role and effectiveness of written information available to patients about individual medicines. Health Technol Assess. 2007;11(5):iii, 1-160.

2. Raynor DK, Svarstad B, Knapp P, et al. Consumer medication information in the United States, Europe, and Australia: A comparative evaluation. J Am Pharm Assoc. 2007;47(6):717-724.

3. Bawazir SA, Abou-auda HS, Gubara OA, et al. Public attitude toward drug technical package inserts in Saudi Arabia. J Pharm Technol. 2003;19:209-218.

4. Alghanim SA. Self-medication practice among patients in a public health care system. East Mediterr Health J. 2011;17(5):409-416.

5. Bin Abdulhak A, Altannir M, Almansor M, et al. Non prescribed sale of antibiotics in Riyadh, Saudi Arabia: a cross sectional study. BMC Public Health. 2011;11:538.

6. Tarn DM, Heritage J, Paterniti DA, et al. Physician communication when prescribing new medications. Arch Intern Med. 2006;166(17): $1855-1862$.

7. Svarstad BL, Bultman DC, Mount JK. Patient counseling provided in community pharmacies: effects of state regulation, pharmacist age, and busyness. J Am Pharm Assoc (2003). 2004;44(1):22-29.

8. Bawazir SA, al-Hassan MI, al-Khamis KI, Abou-Auda HS, Gubara OA. Comparative study of Saudi-marketed products and US drug labeling. DICP. 1991;25(7-8):863-866.

9. Gebran N, Al Haidari K. Assessment of prescribing information for generic drugs manufactured in the Middle East and marketed in Saudi Arabia. Ann Saudi Med. 2006;26(3):192-199.
10. Coulter A. Evidence based patient information is important, so there needs to be a national strategy to ensure it. BMJ. 1998;317(7153): 225-226.

11. Davis TC, Wolf MS, Bass PF, et al. Literacy and misunderstanding prescription drug labels. Ann Intern Med. 2006;145(12):887-894.

12. Wolf MS, Davis TC, Shrank W, et al. To err is human: patient misinterpretations of prescription drug label instructions. Patient Edu Couns. 2007;67(3):293-300.

13. Fuchs J, Hippius M. Inappropriate dosage instructions in package inserts. Patient Educ Couns. 2007;67(1-2):157-168.

14. Berry DC, Knapp P, Raynor DK. Provision of information about drug side-effects to patients. Lancet. 2002;359(9309):853-854.

15. Carrigan N, Raynor DK, Knapp P. Adequacy of patient information on adverse effects: An assessment of patient information leaflets in the UK. Drug Saf. 2008;31(4):305-312.

16. Maat HP, Lentz L. Improving the usability of patient information leaflets. Patient Educ Couns. 2010;80(1):113-119.

17. Shrank W, Avorn J, Rolon C, Shekelle P. Effect of content and format of prescription drug labels on readability, understanding, and medication use: a systematic review. Ann Pharmacother. 2007;41(5):783-801.

18. Pers comm. Ahmed Bahatheq; May 2011.

19. Registered drug list. Saudi Food and Drug Authority. Available from: http://www.sfda.gov.sa/En/Home. Accessed November 2011.

20. Fuchs J, Hippius M, Schaefer M. Analysis of German Package inserts. Int J Clin Pharmacol Ther. 2006;44(1):8-13.

21. Fuchs J. The amount of liquid patients use to take tablets or capsules. Pharmacy Practice (Internet). 2009;7:170-174.

22. Koo M, Krass I, Aslani P. Factors influencing consumer use of written drug information. Ann Pharmacother. 2003;37(2):259-267.

23. Winterstein AG, Linden S, Lee AE, Fernandez EM, Kimberlin CL. Evaluation of consumer medication information dispensed in retail pharmacies. Arch Intern Med. 2010;170(15):1317-1324.

24. Shivkar YM. Clinical information in drug package inserts in India. J Postgrad Med. 2009;55(2):104-107.

25. Zaghi D, Maibach HI. Survey of safety and efficacy information in drug inserts for topical prescription medications. Am J Clin Dermatol. 2007;8(1):43-46.

26. Steinmetz KL, Coley KC, Pollock BG. Assessment of geriatric information on the drug label for commonly prescribed drugs in older people. J Am Geriatr Soc. 2005;53(5):891-894.

27. Haw C, Stubbs J. Patient information leaflets for antidepressants: are patients getting the information they need? J Affect Disord. 2011; 128(1-2):165-170.

28. Luk A, Aslani P. Tools used to evaluate written medicine and health information: document and user perspectives. Health Educ Behav. 2011;38(4):389-403.

29. Guidance for Presenting the SPC, PIL and Labeling information. Saudi Food and Drug Authority. Available from: http://www.sfda.gov.sa/ En/Drug/Topics/Regulations+-+Guidelines.htm. Accessed November 2011.

30. Bunge M, Muhlhauser I, Steckelberg A. What constitutes evidencebased patient information? Overview of discussed criteria. Patient Edu Couns. 2010;78(3):316-328.

31. Schwappach DL, Mülders V, Simic D, et al. Is less more? Patients' preferences for drug information leaflets. Pharmacoepidemiol Drug Saf. 2011;20(9):987-995.
Drug, Healthcare and Patient Safety

\section{Publish your work in this journal}

Drug, Healthcare and Patient Safety is an international, peer-reviewed open-access journal exploring patient safety issues in the healthcare continuum from diagnostic and screening interventions through to treatment, drug therapy and surgery. The journal is characterized by the rapid reporting of reviews, original research, clinical, epidemiological and

\section{Dovepress}

post-marketing surveillance studies, risk management, health literacy and educational programs across all areas of healthcare delivery. The manuscript management system is completely online and includes a very quick and fair peer-review system. Visit http://www.dovepress.com/ testimonials.php to read real quotes from published authors. 